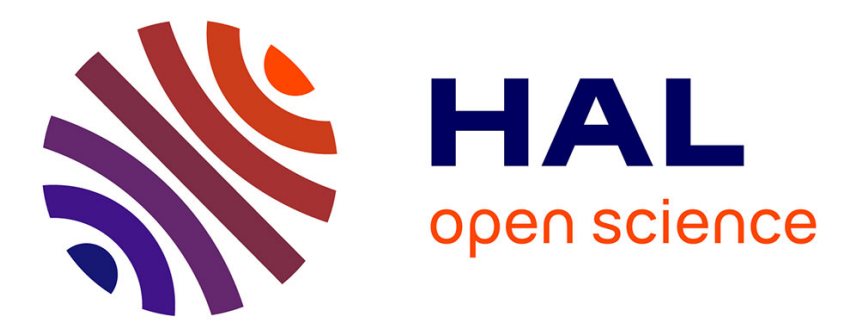

\title{
Virtual implantation and patient-specific simulation for optimization of outcomes in ventricular assist device recipients.
}

Amedeo Anselmi, Sophie Collin, Pascal Haigron, Jean-Philippe Verhoye, Erwan Flecher

\section{To cite this version:}

Amedeo Anselmi, Sophie Collin, Pascal Haigron, Jean-Philippe Verhoye, Erwan Flecher. Virtual implantation and patient-specific simulation for optimization of outcomes in ventricular assist device recipients.. Medical Hypotheses, 2016, 91, pp.67-72. hal-01317440

HAL Id: hal-01317440

https://hal-univ-rennes1.archives-ouvertes.fr/hal-01317440

Submitted on 18 May 2016

HAL is a multi-disciplinary open access archive for the deposit and dissemination of scientific research documents, whether they are published or not. The documents may come from teaching and research institutions in France or abroad, or from public or private research centers.
L'archive ouverte pluridisciplinaire HAL, est destinée au dépôt et à la diffusion de documents scientifiques de niveau recherche, publiés ou non, émanant des établissements d'enseignement et de recherche français ou étrangers, des laboratoires publics ou privés. 
Anselmi et al.

\title{
VIRTUAL IMPLANTATION AND PATIENT-SPECIFIC SIMULATION FOR OPTIMIZATION OF OUTCOMES IN VENTRICULAR ASSIST DEVICE RECIPIENTS
}

\author{
Amedeo ANSELMI ${ }^{\star}$, MD, Sophie COLLIN ${ }^{\S}$, Pascal HAIGRON ${ }^{\S}, \mathrm{PhD}$, \\ Jean-Philippe VERHOYE ${ }^{\star \S}$, MD PhD, Erwan FLECHER ${ }^{\star \S}$, MD PhD \\ *Division of Thoracic and Cardiovascular Surgery, Pontchaillou University Hospital, \\ Rennes, France \\ § INSERM U1099, LTSI Research Unit - Laboratoire Traitement du Signal et de \\ I'Image, University of Rennes 1, Rennes, France
}

Address for correspondence:

Amedeo Anselmi, MD PhDs

Division of Thoracic and Cardiovascular Surgery

Pontchaillou University Hospital

2, rue Henri le Guilloux, 35000 Rennes, France

Tel\# 0033(0)637060379

Fax\# 0033(0)299282497

Email: amedeo.anselmi@alice.it

Sources of Funding: 'Association Ouest Transplant' (Western French Transplantation Association) (partial financial support). 
Anselmi et al.

\section{Abstract}

Background. Left Ventricular Assist Devices (LVAD) are increasingly used for longterm mechanical circulatory support and are effective in improving survival and quality-of-life of patient with advanced heart failure. Nonetheless, they are associated with significant early and late morbidity rates (including pump thrombosis, thromboembolic events, and pump dysfunction). These complications are at least partially associated with suboptimal pump positioning. Currently, we are missing tools to further improve the positioning of LVAD devices in a patient-specific fashion

Methods and evaluation of the hypothesis. We hypothesized that the analysis of the implanted device in patients presenting selected LVAD-related complications through segmentation and three-dimensional reconstruction of CT scans may provide patient-specific information into mechanical factors contributing to pump dysfunction and thromboembolic events, with potential to guide preventive interventions against development of new complications. We also hypothesized that preoperative virtual implantation and computer-assisted surgery in candidates to LVAD implantation may help in the customization of device positioning, with potential to minimize severe complications. The hypothesis was evaluated in a multidisciplinary fashion (cardiac surgeons, biomedical engineers and biomedical images processing experts). CT scans of 14 LVAD recipients were reconstructed through semi-automatic segmentation (including the whole heart, the implanted device and the chest wall). A coordinate system was built to quantify the coaxiality of the LVAD apical cannula with the mitral annulus. Patients were stratified into Group 1 (presenting complications such as thromboembolic events, pump dysfunction or thrombosis) and Group 2 (no complications). Group 1 patients presented significantly greater average rotation of the apical cannula towards the interventricular septum $(p=0.015)$, although no difference was observed in terms of average rotation towards the anterior or posterior left ventricular wall.

Conclusions. Several patient-specific factors (including left ventricular morphology and chest wall conflict with the device after wound closure) may influence the effectiveness and safety of LVAD therapy, but they are difficultly managed through the current implantation techniques. We suggest that the clinical results of LVAD 
Anselmi et al.

treatment can be improved through preoperative virtual implantation and computerassisted surgery (in order to guide device selection, exact site of left ventricular wall coring site). Given these preliminary results, we are examining larger patient datasets in order to further test the hypothesis. Dedicated tools for virtual implantation are currently under development.

KEY WORDS: Left Ventricular Assist Device; Computer-assisted surgery; Clinical outcomes 
Anselmi et al.

\section{Introduction}

Although heart transplantation (HTx) remains the treatment of choice for advanced heart failure (HF), the worsening shortage of donors worldwide represents the major limitation of this therapy. In such scenario, the employment of long-term mechanical circulatory support has gained increasing importance in the current practice. The current implantable continuous-flow left ventricular assist devices (LVADs), based on magnetically-driven rotors and external power source, and are characterized by improved durability and smaller size than previous pulsatile intra- or paracorporeal devices [1]. Generally speaking, LVADs include an inflow cannula surgically placed at the left ventricular (LV) apex for drainage of blood, a pump body containing the propelling rotor, and an outflow conduit driving the propelled blood into the ascending aorta (Figure 1). Power is provided by a driveline exiting the skin usually in the abdominal region and connected to a controller with batteries. Continuous-flow LVADs have been associated with a decrease in the rate of device-related complications and better clinical outcomes, including survival time on support $[2,3]$. LVADs can be employed either as a bridge to heart transplantation (BTT) or as a destination therapy (DT) in patients who are ineligible to transplant. LVADs represent the vast majority of the employed long-term Mechanical Circulatory Support (MCS) devices [4], over biventricular support strategies and pulsatile devices. Nonetheless, LVAD therapy remains associated with significant rates of major morbidity, both immediate and in the long-term follow-up. These include mainly driveline and pump pocket infection, right ventricular failure, thromboembolic and hemorrhagic events, pump thrombosis, and rhythm disturbances [5]. A number of these complications have been associated with suboptimal LVAD implantation and positioning of LVAD components with respect to the cardiac structures [6]. 
Anselmi et al.

The implantation procedure of LVADs has been based so far on general anatomical principles and surgeon's adaptation to the individual anatomical characteristics, on the basis of clinical common sense. Similarly, the pathophysiology of many cases of pump thrombosis and thromboembolic events during LVAD support remains unknown $[4,7]$. These complications represent a growing problem during the recent years [8]. The quality of apical cannula position, the occurrence of stasis and turbulent blood flow within the LV cavity as well as within the aortic root represent major determinants of thrombotic events $[6,7]$. Currently, we are missing tools to further improve the positioning of LVAD devices in a patient-specific fashion.

\section{The Hypothesis}

We postulated that:

1. In patients presenting selected complications under LVAD support (i.e. thromboembolic events, pump thrombosis, severe rhythm disturbances, ineffective LV drainage, etc.), the analysis of the implanted LVAD through segmentation and three-dimensional reconstruction of the patients' CT scan may provide patient-specific insights into the pathophysiology of the complication. Therapeutic interventions (i.e. adaptation of the anticoagulant therapy) could be prompted as a consequence.

2. In candidates to LVAD treatment, preoperative virtual implantation and subsequent computer-assisted surgery might enhance the implantation of LVAD in a patient-specific fashion. Optimization of LVAD function and 
Anselmi et al.

decrease in the rate of both immediate and long-term complications are expected.

\section{Evaluation of the Hypothesis}

For the preliminary testing of the hypothesis, we assembled a dedicated research team combining heart failure surgeons (AA and EF), biomedical engineers (SC) and an expert in medical image processing for innovative clinical applications ( $\mathrm{PH})$ [9]. We retrospectively selected patients who received LVAD implantation at a single University cardiac surgery center, and who had undergone contrast-enhanced chest CT scan during the follow-up. The indication to LVAD therapy was decided by multidisciplinary consensus of heart failure cardiologist and cardiac surgeons, according to the current recommendations [10]. CT scans had to be performed with a slice thickness $\leq 1.25 \mathrm{~mm}$ with contrast injection of both ventricles and atria; they had to present limited noise and artifacts due to other endovascular devices (i.e., pacemaker and internal automatic defibrillator leads, although the presence of any of these devices did not constitute an exclusion criterion) and sufficient visibility of the mitral valve leaflets and annulus (grey values calibrated on the Hounsfield scale). According to these criteria, among 47 consecutive patients who received LVAD implantation at our center during the 2008-2014 period, we retrospectively selected 14 individuals (BTT in 9 cases and DT in 5 cases) having postoperative CT scan complying with the above requirements. CT scan investigations were performed during the follow-up on the basis of various indications (such as exploration of driveline infection and ruling out of intracavitary thrombus). The implanted LVAD was the Thoratec HeartMate II in 10 instances (Thoratec Corporation, Pleasanton, CA), 
Anselmi et al.

the Jarvik2000 Flowmaker in 3 cases (Jarvik Heart Inc., New York, NY), and the HVAS in one (Heartware Inc., Framingham, MA). CT scans were elaborated in order to achieve a three-dimensional reconstruction of the whole heart, of the LVAD and of the thoracic wall (osseous components) through semi-automatic segmentation method (ITK-Snap software, Philadelphia, PA).

The postoperative analysis of device positioning was based on the principles of the state-of-the-art implantation, as recommended by the consensus of LVAD surgeons and manufacturers' instructions [6]. The intraventricular inflow cannula needs to be coaxial with the mitral valve orifice and parallel to the interventricular septum, in order to optimize blood drainage and avoid conflict of the cannula with the endocardial surface. This may lead to obstructed flow, suboptimal device functioning, ventricular arrhythmias and potentially to thromboembolic events due to blood stasis and turbulences. In order to calculate the coaxiality with the mitral valve orifice, users (LVAD surgeons) were asked to define on postoperative CT scan slices a series of points (between 15 and 20) corresponding to the contour of the mitral annulus (3D Slicer Software [11]). The interface subsequently provides the average plane of the mitral valvular orifice and the center of the orifice (Figure 2A). A coordinate system was defined in order to quantify the coaxiality of the implanted LVAD's apical cannula with this plane. Briefly, the latero-lateral axis $(x)$ is defined by the center of the orifice and the annular point corresponding to the anterior mitral commissure, and the anterior-posterior axis $(\mathrm{y})$ is defined as the perpendicular to the latero-lateral axis on the mitral plane. The third axis $(z)$ is defined as the perpendicular to the mitral plane at its center. Subsequently, the axis of the LVAD apical cannula is obtained from the representation of the implanted device from semi-automatic segmentation. The axis of the apical cannula is described with respect to the mitral valve coordinates system 
Anselmi et al.

according to two angles: $\varphi$ (representing the orientation of the LVAD cannula with respect to the anterior or inferior LV wall) and $\theta$ (representing the orientation of the LVAD cannula towards either the lateral LV wall or the interventricular septum - IVS) (Figure 2B). These angles provide the rotation coordinates of the LVAD apical cannula with respect to the mitral valve orifice. Ideal values are $\varphi=0^{\circ}$ and $\theta=0^{\circ}$. For the $\theta$ angle, values $>0^{\circ}$ and $<0^{\circ}$ indicate displacement towards the anterior or inferior LV wall, respectively. For the $\varphi$ angle, values $>0^{\circ}$ and $<0^{\circ}$ indicate displacement towards the lateral LV wall or the IVS, respectively. Angles $\varphi$ and $\theta$ were calculated for the 8 patients included in this preliminary study; values could be also expressed as percent variation with respect to the ideal value. This percentage is expressed with a based number of $90^{\circ}\left(\varphi=0^{\circ}\right.$ represents a variation of $0 \%, \varphi=+90^{\circ}$ or $\varphi=-90^{\circ}$ represent a variation of $100 \%)$.

The patients' clinical records were reviewed in order to identify early postoperative and late complications which could be ascribed to suboptimal device positioning. Among these, we included thrombotic and thromboembolic events (pump thrombosis, LV cavity thrombus, stroke, transient ischemic attack - TIA, suboptimal pump flow and persisting suction events despite intravascular volume expansion, severe early ventricular arrhythmias after LVAD implantation in patients without preoperative history of severe arrhythmia). Suction events are characterized by iterative, abrupt drops in pump flow due to impingement of the apical cannula orifice with the endocardial surface (typically, the IVS). Severe repeated ventricular arrhythmias immediately after LVAD implantation can be ascribed to inappropriate stimulation of the endocardial surface from a misplaced cannula. Hemorrhagic stroke was not considered as a complication endpoint with respect to the present investigation, similar to other issues such as noncerebral hemorrhagic events and driveline/pump 
Anselmi et al.

pocket infection. These were considered to be scientifically unrelated with the purposes of this study. We stratified the patients according to the history of at least one of these events within either the Complicated (Group 1) or Uncomplicated (Group 2) subgroup. Adverse events on LVAD support were defined according to current multi-Institutional registries [12].

\section{Analysis and data management.}

Data were included prospectively into an electronic database. Average deviation of apical cannula axis with respect to the ideal value was described as mean \pm standard deviation (continuous data). Categorical data were presented as percentages. Continuous data were compared using the two-tailed Student's $t$ test. The Kolgoromov-Smirnoff test was employed to verify normality of distribution. The alpha value was 0.05 ; analyses were performed through the SPSS software ver. 19.0 for Windows (SPSS, Chicago, IL), Sample size calculation was conducted using the PS software ver. 3.0 for Windows.

The present retrospective study did not entail any additional diagnostic or therapeutic protocol than current clinical management, and all data were managed anonymously. 
Anselmi et al.

\section{Empirical data}

The segmentation of the whole heart and of the implanted LVAD could be successfully performed for all of the selected patients. Table 1 summarizes the results of the analysis of apical cannula axis positioning with respect to the mitral annular plane, stratified among complicated vs. uncomplicated patients. Seven patients $(50 \%$ of the present population) were included in the Complicated subgroup (Group 1) and the remainders entered the Uncomplicated subgroup (Group 2). Complications were thromboembolic events (stroke and TIA) in four cases, pump thrombosis in one case and device malfunction (device failure and early severe ventricular arrhythmias) in two cases. Average duration of postoperative follow-up was 24.9 months \pm 16.1 and 29.4 months \pm 13.7 in the Complicated and Uncomplicated Group, respectively $(p=0.53)$. In Group 1, all but two patients presented rotation of the apical cannula towards the interventricular septum (IVS). For these two cases, percent rotation towards the left ventricular lateral free wall was minimal (average: $4.4 \%$ ). Average percent rotation of the apical cannula towards the IVS was $55.1 \% \pm 31.8$ in Group 1 , and $13 \% \pm 12.1$ in Group $2(p=0.015)$. Average percent rotation of the apical cannula towards the inferior or anterior LV wall was $36 \% \pm 26.4$ in Group 1, and 46.8\% \pm 28.8 in Group $2(p=0.48)$.

Reconstruction and morphological examination of CT scans of LVAD recipients suggested the role of device contact with the thoracic wall (closed chest conditions) 
Anselmi et al.

in determining device and cannula displacement with respect to the target position (Figure $3 \mathrm{~A}$ and $3 \mathrm{~B}$ ).

A sample size calculation suggested that a total of 154 patients should be enrolled to one among two alternative strategies (with or without postoperative analysis of device positioning and consequent management of anticoagulant treatment) to detect with a $80 \%$ power and a 0.05 alpha level a significant $50 \%$ reduction in the rate of thromboembolic events during a 3-years follow-up. This sample size calculation will need to be refined through data from additional preliminary investigations. 
Anselmi et al.

\section{Consequences of the Hypothesis}

Landmark randomized studies have demonstrated that LVAD implantation is associated with better survival than medical treatment in patients with end-stage heart failure who are not candidates to heart transplantation (REMATCH Study) [13]. LVAD implantation also allows improved functional capacities and provides a better quality of life to these patients $[14,15]$. Currently, around 4,000 new patients are enrolled every year in the international INTERMACS registry after having received an LVAD [4]. Moreover, the proportion of patients receiving LVAD as DT is constantly increasing over BTT patients; this amplifies the need to further reduce the complications rate under support for patients who cannot benefit of heart transplant in case of LVAD-related issues. The occurrence of complications during LVAD support (namely, thrombotic and embolic events) is multifactorial, and proceeds from the interaction of multiple patient- and device-related elements. Mechanical and nonmechanical factors (including suboptimal anticoagulant therapy and proinflammatory conditions) can be involved in the pathophysiology of thrombotic events. The present work is specifically addressed at the prevention of mechanical causes through improved implantation of LVADs. There is a link between suboptimal device implantation and pump thrombosis or thromboembolic events [16, 17]. It has been suggested that the inflow cannula of an HeartMate II LVAD should present an angle of approximately $15^{\circ}-30^{\circ}$ with respect to the patient's vertical axis and of $75^{\circ}-60^{\circ}$ relative to the pump body on anteroposterior chest X-rays. The apical cannula should 
Anselmi et al.

also be parallel to the IVS [6]. The site of apical LV coring plays a major role in the determinism of cannula orientation. Nonetheless, the rate of stroke under LVAD support remains considerable despite accurate surgical technique. Although the rate of thrombotic and thromboembolic complications on LVAD support is relatively contained, their occurrence is associated with major morbidity/mortality and loss of quality of life. Therefore, their prevention is among the main concerns of LVAD teams.

We hypothesized that more refined analysis of pump positioning is required to better understand the propensity to thrombosis and suboptimal device function in individual patients, and that the implantation technique can be further improved and customized through preoperative virtual implantation and computer-assisted surgery. Such hypothesis is supported not only by previous literature and clinical experience, but also by the present original data suggesting that less proper intraventricular cannula orientation is associated with occurrence of early and late complications. In particular, Group 1 patients showed significantly greater percent improper cannula rotation towards the IVS $(\mathrm{p}=0.015)$. In the present limited series, we did not observe a significant difference in terms of average percent $\theta$ rotation among subgroups. Nonetheless, average $\varphi$ rotation towards the IVS was significantly different among the complicated vs. uncomplicated patients groups, and it may become a useful indicator when markedly oriented towards the IVS. Given the present data, we will expand the dataset through performance of dedicated CT scan of additional patients in order to gather additional information on these issues. Although the presence of intracavitary defibrillator and pacemaker leads may generate artifacts, this only occasionally compromises the exploitability of CT-scan images with the purposes of $3 \mathrm{D}$ reconstruction and device positioning analysis. Future developments will involve 
Anselmi et al.

the inclusion of flow simulation within reconstructed LV cavities of patients under LVAD. Several previous works employing numerical hemodynamic simulation have suggested a link between the morphology of the LV cavity, the position of the LVAD inflow cannula and the development of flow patterns predisposing to thrombus formation $[18,19,20]$. Nonetheless, no clinical application has been attempted until now.

During current state-of-the-art LVAD implantation, the surgeon identifies the LV wall coring zone within a region at the LV apex lateral to the left anterior descending artery, under transesophageal echocardiography guidance to try achieving coaxiality with the mitral valve orifice. Such protocol remains essentially qualitative and exposes to the risk to disregard individual anatomical features with potential impact on final placement of the apical cannula. In fact, LVAD recipients present a vast array of underlying etiologies (primary dilated cardiomyopathy, ischemic heart disease, hypertrophic cardiomyopathy...) with variable degrees of symmetric or asymmetric LV dilatation and different morphologies of the LV cavity [4]. This determines an extreme variability in intracavitary geometrical and flow conditions after LVAD implantation. Additionally, since LVADs are implanted through full median sternotomy in the vast majority of instances, it is difficult for the operating surgeon to anticipate the interaction between the chest wall and the device after chest closure. Implantation of the LVAD cannula demands luxation of the LV apex and distortion of the normal anatomical relations. This adds another factor of variability with respect to the choice of the cannulation site and the final positioning of the LVAD device within the chest. Device conflict with the rigid chest wall may alter the apical cannula orientation at the end of surgery. Until now, the latter aspect has been investigated in the field of total artificial heart implantation [21]. Herein, original analysis of the 
Anselmi et al.

interaction between the closed chest wall and the implanted device (Figure 3) provides one potential explanation for postoperative device migration [6], as well as one further variable to be considered in the computer-assisted planning of LVAD implantation.

Computer-assisted surgery is a complex discipline integrating various technologies and sources of data, such as pre- and intraoperative imaging, virtual reality, augmented reality, interactive simulation and micro-technologies $[9,22]$. The major fields of application have been neurosurgery and orthopedic surgery [23, 24, 25], although innovative applications are being proposed in endovascular surgery [26]. The present preliminary work in the field of surgery for advanced heart failure opens new avenues of potential applications, including preoperative planning of optimal LVAD positioning (i.e., epicardial coring site which allows the best possible alignment with the mitral valve and less generation of intracavitary turbulent flow patterns, taking into account the geometrical limitations imposed by the chest wall).

Henceforth, we started the development of a tool for preoperative virtual implantation for intended future clinical use. Segmentation and reconstructed cardiac volumes were managed through a computer interface (Figure 4A). In such interface, the reconstructed cardiac structures can be scrolled within a 3D slice view corresponding to the representation of the coronal, transversal and sagittal CT scan slices. Parallel to cardiac chamber segmentation, we constructed a three-dimensional mesh component representation of two commercially available LVADs (ITK-Snap software, Philadelphia, PA). The Thoratec HeartMate II and the HeartWare HVAS were employed. To such purpose, we used rotational imaging of the LVADs (conebeam computed tomography) in order to minimize artifacts (Figure 4B). The mesh representation of LVADs can be integrated within the same interface in order to allow 
Anselmi et al.

real-time spatial manipulation by the user and virtual implantation within the CT scan volumes (Figure 4C). Our ultimate purpose is to provide a user-friendly software for interactive simulation and procedural planning, which could be employed by surgeons and cardiologists. Other software are currently used clinically for, in example, sizing transcatheter heart valves and aortic endoprostheses. Such developments proceed from the hypothesis illustrated herein and from the above empirical data. These developments are still at an early stage; nevertheless, verification of the hypothesis through analysis of further postoperative CT-scans will be required.

There are several limitations to the present investigations, including the limited number of patients contributing to the empirical data, and the purely morphological approach employed so far in the analysis of implanted patients. Despite these limitations, we can hypothesize that the current clinical results of LVAD treatment can be optimized through preoperative computer-assisted simulation of device implantation. In patients who have been already implanted, screening through CT scan and apical cannula position analysis (coupled with intracavitary flow pattern simulation) may help in the future to stratify the propensity to develop thromboembolic complications and guide preventive intervention (adaptation of the anticoagulant therapy and addition of long-term antiplatelet treatment). Such hypothesis is supported by original data. Residual LV volume around the inflow cannula and its distance from the mitral valve plane will also be investigated for relationship with adverse events. Additionally, the morphology of the inflow cannula varies among the commercially available LVADs. This will need to be taken into account into future studies, through achievement of sufficient sample size to stratify the results according to devices, and through analysis of the hemodynamic effects of 
Anselmi et al.

various cannula morphologies. On these bases, we are going to initiate a prospective study in larger patient subsets to validate such hypothesis. Multicentre cooperation will be probably necessary at more advanced research stages in order to clinically demonstrate a reduction in thromboembolic rate. Preoperative virtual implantation and subsequent computer-assisted surgery strategies may be employed in the future to achieve a greater and customized level or precision and reproducibility in LVAD implantation. 
Anselmi et al.

\section{Acknowledgements}

The Authors are grateful to the 'Association Ouest Transplant' (Western French Transplantation Association) for partially funding this work.

This work has been partially conducted in the experimental platform TherA-Images (Rennes, France) supported by Europe FEDER

\section{References}

1. Slaughter MS, Pagani FD, Rogers JG et al. Clinical management of continuous-flow left ventricular assist devices in advanced heart failure. $J$ Heart Lung Transplant. 2010;29(4 Suppl):S1-39.

2. Miller LW, Pagani FD, Russell SD et al. Use of a continuous-flow device in patients awaiting heart transplantation. N Engl J Med. 2007;357:885-96.

3. Pagani FD, Miller LW, Russell SD et al. Extended mechanical circulatory support with a continuous-flow rotary left ventricular assist device. J Am Coll Cardiol. 2009;54:312-21.

4. Kirklin JK, Naftel DC, Pagani FD et al. Sixth INTERMACS annual report: a 10,000-patient database. J Heart Lung Transplant. 2014;33:555-64. 
Anselmi et al.

5. Puehler T, Ensminger S, Schoenbrodt M et al. Mechanical circulatory support devices as destination therapy-current evidence. Ann Cardiothorac Surg. 2014;3:513-24.

6. Adamson RM, Mangi AA, Kormos RL, Farrar DJ, Dembitsky WP. Principles of HeartMate II implantation to avoid pump malposition and migration. J Card Surg. 2015;30:296-9.

7. Blitz A. Pump thrombosis-A riddle wrapped in a mystery inside an enigma. Ann Cardiothorac Surg. 2014;3:450-71.

8. Starling RC, Moazami N, Silvestry SC et al. Unexpected abrupt increase in left ventricular assist device thrombosis. N Engl J Med. 2014;370:33-40.

9. Haigron P, Dillenseger JL, Luo L, Coatrieux JL. Image-guided therapy: evolution and breakthrough. IEEE Eng Med Biol Mag. 2010;29:100-4.

10. Feldman D, Pamboukian SV, Teuteberg JJ et al. The 2013 International Society for Heart and Lung Transplantation Guidelines for mechanical circulatory support: executive summary. J Heart Lung Transplant. 2013;32:157-87.

11. Fedorov A, Beichel $R$, Kalpathy-Cramer $J$ et al. 3D Slicer as an image computing platform for the Quantitative Imaging Network. Magn Reson Imaging. 2012;30:1323-41.

12. Kirklin JK, Naftel DC, Kormos RL et al. Fifth INTERMACS annual report: risk factor analysis from more than 6,000 mechanical circulatory support patients. J Heart Lung Transplant. 2013;32:141-56.

13. Rose EA, Gelijns AC, Moskowitz AJ et al. Long-term use of a left ventricular assist device for end-stage heart failure. N Engl J Med. 2001;345:1435-43. 
Anselmi et al.

14. Carrel T, Englberger L, Martinelli MV et al. Continuous flow left ventricular assist devices: a valid option for heart failure patients. Swiss Med Wkly. 2012;142:w13701.

15. Jorde UP, Kushwaha SS, Tatooles AJ et al. Results of the destination therapy post-food and drug administration approval study with a continuous flow left ventricular assist device: a prospective study using the INTERMACS registry (Interagency Registry for Mechanically Assisted Circulatory Support). J Am Coll Cardiol. 2014;63:1751-7.

16. Taghavi S, Ward C, Jayarajan SN et al. Surgical technique influences HeartMate II left ventricular assist device thrombosis. Ann Thorac Surg. 2013;96:1259-65.

17. Uriel N, Han J, Morrison KA et al. Device thrombosis in HeartMate II continuous-flow left ventricular assist devices: a multifactorial phenomenon. $J$ Heart Lung Transplant. 2014;33:51-9.

18. Fraser KH, Zhang T, Taskin ME, Griffith BP, Wu ZJ. Computational fluid dynamics analysis of thrombosis potential in left ventricular assist device drainage cannulae. ASAIO J. 2010;56:157-63.

19. Ong C, Dokos S, Chan B et al. Numerical investigation of the effect of cannula placement on thrombosis. Theor Biol Med Model. 2013;10:35.

20. McCormick M, Nordsletten D, Kay D, Smith N. Modelling left ventricular function under assist device support. Int J Num Methods Biomed Eng 2011;27: 1073-1095.

21. Moore RA, Madueme PC, Lorts A, Morales DL, Taylor MD. Virtual implantation evaluation of the total artificial heart and compatibility: Beyond standard fit criteria. J Heart Lung Transplant. 2014;33:1180-3. 
Anselmi et al.

22. Peters TM. Image-guidance for surgical procedures. Phys Med Biol. 2006;51:R505-40.

23. D'Amico RS, Kennedy BC, Bruce JN. Neurosurgical oncology: advances in operative technologies and adjuncts. J Neurooncol. 2014;119:451-63.

24.Wang LL, Leach JL, Breneman JC, McPherson CM, Gaskill-Shipley MF. Critical role of imaging in the neurosurgical and radiotherapeutic management of brain tumors. Radiographics. 2014;34:702-21.

25. Goradia VK. Computer-assisted and robotic surgery in orthopedics: where we are in 2014. Sports Med Arthrosc. 2014;22:202-5.

26. Kaladji A, Lucas A, Cardon A, Haigron P. Computer-aided surgery: concepts and applications in vascular surgery. Perspect Vasc Surg Endovasc Ther. 2012;24:23-7. 
Anselmi et al.

\section{Figure Legends}

Figure 1. Representation of a Thoratec HeartMate II LVAD.

Figure 2. Analysis of apical LVAD inflow cannula position with respect to the target mitral valve orifice (green disks). A. The mitral valve plane is defined semiautomatically by the user. B. Coordinates system to describe the orientation angles $(\varphi$ and $\theta)$.

Figure 3. Analysis of LVAD interaction with the thoracic wall in closed chest conditions. A. Reconstruction of post-implantation CT scan showing anterior displacement of the intraventricular inflow cannula. B. Addition of the chest wall structure reconstruction in the same patient showing thoracic collision and consequent posterior orientation of the pump body.

Figure 4. Development of an interactive interface for CT scan navigation, segmentation of cardiac chambers and manipulation of LVAD component mesh. A. Cardiac volumes segmentation. B. Component mesh representation of a HeartMate II LVAD. Nc is the vector representing the inflow cannula axis. C. Example of spatial manipulation of the LVAD component mesh by the user within the $3 \mathrm{D}$ environment. 
Anselmi et al.

Table 1. Analysis of positioning of LVAD apical cannula with respect to the mitral valve plane. Group 1 and 2 are the Complicated and Uncomplicated subgroups, respectively. IVS: Interventricular septum. See the text for definitions. *Modified interpretation of angles due to adaptation of the coordinate system to the anatomy of this particular case.

\begin{tabular}{|c|c|}
\hline Patient & Orientation $\left({ }^{\circ}\right)$ \\
\hline P4 (Group 1) & $\begin{array}{c}\theta=-41.17(45.7 \% \text { inferior }) \\
\varphi=-82.54(91.7 \% \text { towards IVS) }\end{array}$ \\
\hline P10 (Group 1) & $\begin{array}{c}\theta=72.40(80.4 \% \text { anterior }) \\
\varphi=-35.98(40 \% \text { towards IVS })\end{array}$ \\
\hline P14* (Group 1) & $\begin{array}{l}=78.48(87.2 \% \text { towards IVS }) \\
\varphi=24.04(26.7 \% \text { anterior })\end{array}$ \\
\hline P16 (Group 1) & $\begin{array}{c}\theta=51.78(57.5 \% \text { anterior }) \\
\varphi=6.86(7.63 \% \text { lateral })\end{array}$ \\
\hline P17 (G & $\begin{array}{c}\theta=9.66(10.7 \% \text { anterior }) \\
\varphi=-27.6(30.7 \% \text { towards IVS })\end{array}$ \\
\hline PJ3 (Group 1) & $\begin{array}{c}\theta=-19.2(21.3 \% \text { inferior }) \\
\varphi=1.02(1.13 \% \text { lateral })\end{array}$ \\
\hline PW1 (Group 1) & $\begin{array}{c}\theta=51.78(57.5 \% \text { anterior }) \\
\varphi=6.86(7.63 \% \text { lateral })\end{array}$ \\
\hline P3 (Group 2) & $\begin{array}{c}\theta=-8.62(9.58 \% \text { inferior }) \\
\varphi=-23.36(25.9 \% \text { towards IVS })\end{array}$ \\
\hline P5 (Group 2) & $\begin{array}{c}\theta=37.47(41.6 \% \text { anterior }) \\
\varphi=-9.74(10.8 \% \text { towards IVS })\end{array}$ \\
\hline P9 (Group 2) & $\begin{array}{c}\theta=53.76(59.7 \% \text { anterior }) \\
\varphi=-2.36(2.6 \% \text { towards IVS })\end{array}$ \\
\hline
\end{tabular}


Anselmi et al.

P13 (Group 2)

P15 (Group 2)

PJ1 (Group 2)

PJ2 (Group 2) $\theta=82.56(91.7 \%$ anterior $)$

$\varphi=-32.09(35.6 \%$ towards IVS)

$\theta=51.7$ (57.4\% anterior)

$\varphi=-13.54$ (15\% towards IVS)

$$
\theta=-12.02(13.4 \% \text { inferior })
$$$$
\varphi=1.49 \text { (1.66\% lateral) }
$$

$\theta=7.89$ ( $8.8 \%$ anterior)

$\varphi=-2.54(2.82 \%$ towards IVS) 
Anselmi et al.

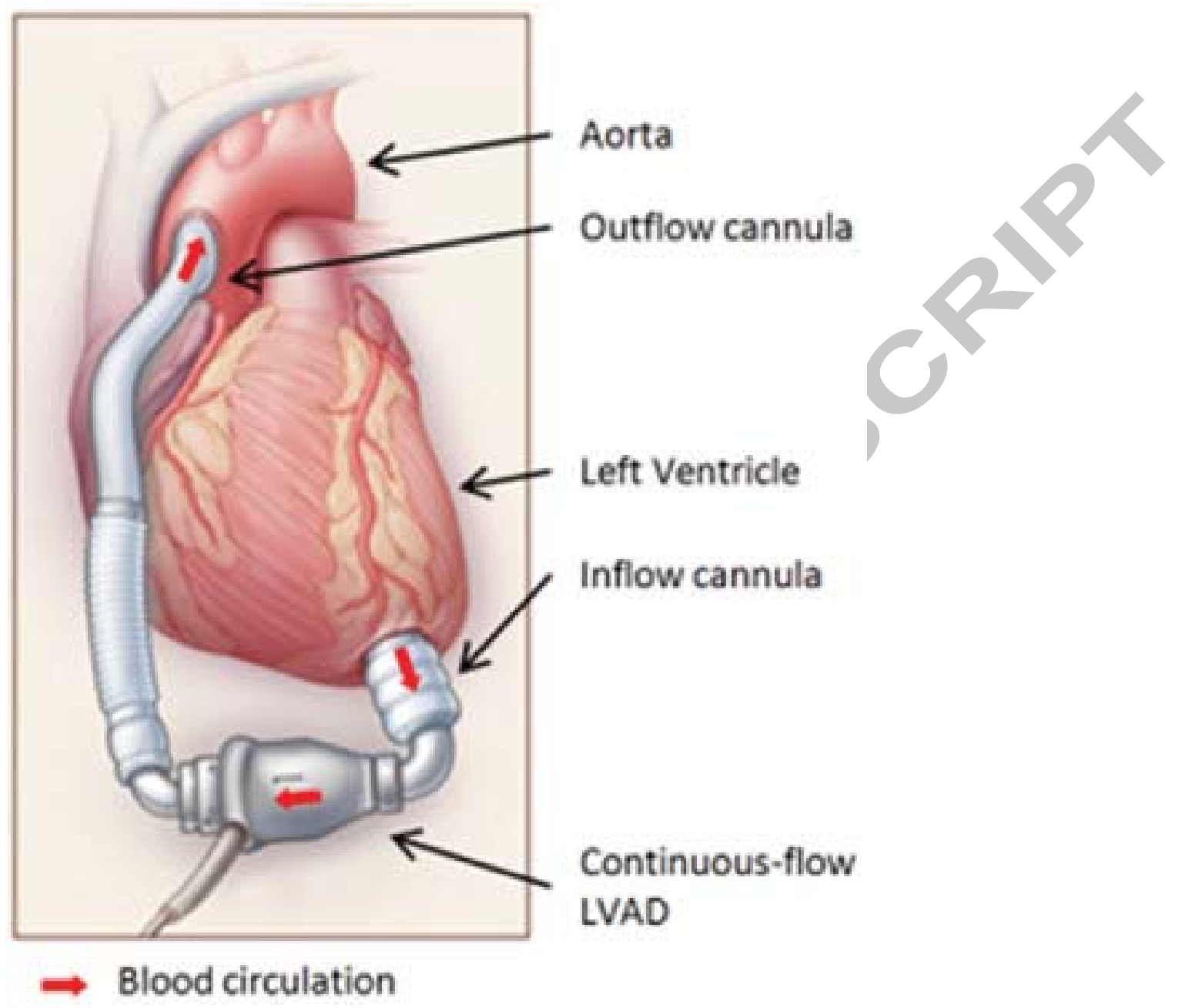




\section{ACCEPTED MANUSCRIPT}

Anselmi et al.

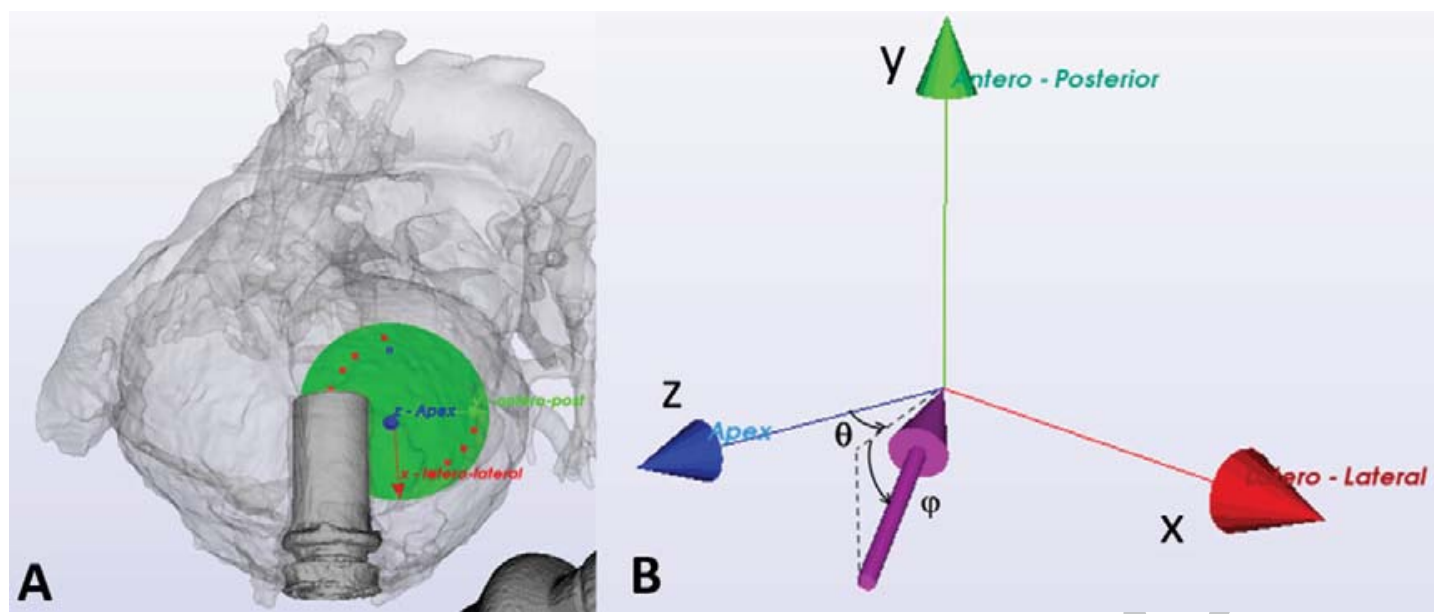




\section{ACCEPTED MANUSCRIPT}

Anselmi et al.
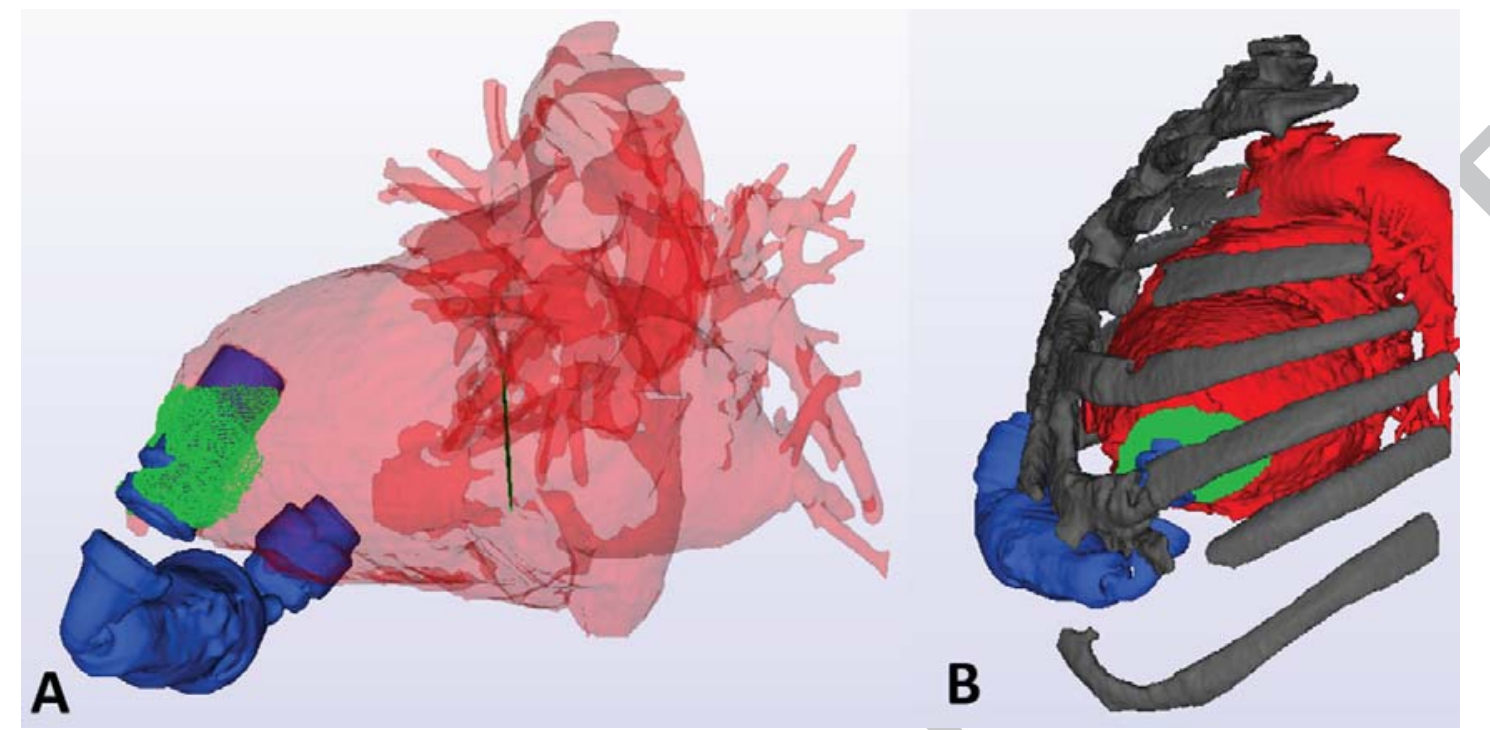
Anselmi et al.
